\title{
Efficacy Assessment of Emergency Percutaneous Coronary Interven- tion (PCI) for Acute Myocardial Infarction (AMI)
}

\author{
Jiajuan Chen*, Hezhong Zhu, Jing Xu, Zhen Gao, Fei Cheng, Yunjie Wei
}

Taihe Hospital in Shiyan City, Affiliated Taihe Hospital of Hubei University of Medicine, Shiyan 442000, Hubei proince, China. E-mail: 769301593@qq.com

\begin{abstract}
Objective: To assess the clinic effect of percutaneous coronary intervention in the treatment of acute myocardial infarction. Methods: 90 patients with acute myocardial infarction in our hospital were chosen to be research objects and they were divided into two groups: control group and research group. Patients in control group were only treated by thrombolytic therapy while those in research group were further treated by percutaneous coronary intervention on the basis of this treatment. Result: The efficacy of research group was higher than that in control group. The incidence of adverse events was $4.44 \%$, which is lower than that in control group. Conclusion: We should effectively apply percutaneous coronary intervention in treating acute myocardial infarction so as to improve the cardiac function of the patients. In addition, this treatment is safer and will lower the incidence of heart and renal failure.
\end{abstract}

Keywords: Acute Myocardial Infarction; Thrombolytic Therapy; Percutaneous Coronary Intervention

\section{Introduction}

Acute myocardial infarction is a very common severe case in clinic treatment. It frequently occurred in the mid-aged and the elderly group. When patients were attacked by such disease, there is always a significant higher level of myocardial enzyme series in their bodies, which will badly affect their daily life. With reference to the clinic treatment of myocardial infarction and a further exploration on this issue, we find that the efficacy is significantly raised when applying percutaneous coronary intervention in the treatment, which will highly improve the patients' health conditions by raising their cardiac function and will also lower the case fatality.

\section{Data and methods}

\subsection{Data}

From March 2017 to March 2019, 90 patients with acute myocardial infarction in our hospital were chosen to be research objects and they were divided into two groups: control group and research group ${ }^{[1]}$. Among the 45 patients in control group, there are 28 male patients and 17 female patients with an average age of 58.76 \pm 7.04 . And among the 45 patients in research group, 26 are male and 19 are female, with an average age of $57.47 \pm 6.34$. Since there was no significant difference in data between the two groups, the data are comparable.

\subsection{Methods}

Patients in control group were treated by thrombolytic therapy, that is to say, they were treated by intravenous infusion by giving 500,000 units of urokinase when diluted to 1 million units and mixed with $100 \mathrm{ml}$ of normal saline ${ }^{[2]}$. However, patients in research group will be further treated by percutaneous coronary intervention on the basis of this treatment. Detail treatments are as follows: firstly, transferring patients and then treating them by percutaneous coronary intervention: patients who are not suitable for thrombolytic therapy should be

This is an open-access article distributed under the terms of the Creative Commons Attribution Non-Commercial License (http://creativecommons.org/licenses/by-nc/4.0/), which permits unrestricted non-commercial use, distribution, and reproduction in any medium, provided the original work is properly cited. 
transferred to hospitals with percutaneous coronary intervention $^{[3]}$. Secondly, directly treating patients by percutaneous coronary intervention: to further dredge infraction artery 12 hours before the outbreak and the treatment time shall be controlled within 90 minutes.

\subsection{Observation index and assessment standard}

\subsubsection{Observation index}

To figure out and compare the incidence of adverse events and the changes of patients' cardiac function level before and after the treatment.

\subsubsection{Efficacy assessment standard}

Excellent Efficacy: the patients' conditions of coronary artery stenoses significantly improved after treatment; Average efficacy: the patients don't occur angina and have no adverse reaction after treatment; Naught efficacy: there are no improvement in acute myocardial infarction or the patients died after treatment ${ }^{[4]}$.

\subsection{Statistical methods}

Statistic software SPSS22.0 is used to analyze the data. The measurement data are expressed as " $\mathrm{x} \pm \mathrm{s}$ ” and will be testified by $t$. The adoption rate of enumeration data is expressed by $\%$ and is testified by $\mathrm{X}^{2} . \quad \mathrm{P}<0.05$ represents the difference. When the probability of the difference is below 0.05 , the data bears statistical significance.

\section{Results}

\subsection{Comparison of incidence of adverse events between two groups}

The incidence of adverse events in research group is $4.44 \%$, lower than $17.78 \%$ in control group. And this difference bears great statistical significance $(\mathrm{P}<0.05)$. See in Table 1.

Table 1. The incidence of adverse events in two groups [n(\%)]

\begin{tabular}{llllll}
\hline Groups & Cases & Hemmorrhage & Renal failure & Angina & Incidence \\
\hline Research Group & 45 & 0 & $1(2.22)$ & $1(2.22)$ & $2(4.44)$ \\
Control Group & 45 & $3(6.67)$ & $3(6.67)$ & $3(6.67)$ & $9(20.00)$ \\
$\mathrm{X}^{2}$ & & & & & 4.050 \\
$\mathrm{p}$ & & & & & 0.044 \\
\hline
\end{tabular}

\subsection{Comparison of Clinic efficacy between two groups}

The total efficacy in research group is higher than that in control group and the difference bears great statistical significance $(\mathrm{P}<0.05)$. See in Table 2.

Table 2. Comparison of Clinic efficacy between two groups

\begin{tabular}{llllll}
\hline Groups & Cases & Excellent efficacy & Average efficacy & Naught efficacy & Total efficacy \\
\hline Research Group & 45 & $20(44.44)$ & $24(53.33)$ & $1(2.22)$ & $44(97.78)$ \\
Control Group & 45 & $18(40.00)$ & $20(44.44)$ & $7(15.56)$ & $38(84.44)$ \\
$X^{2}$ & & & & & 4.939 \\
p & & & & 0.026 \\
\hline
\end{tabular}

\section{Discussion}

Acute myocardial infarction is characterized by severity and urgency in outbreak. It is usually caused by an emergency block in coronary artery in patients' body ${ }^{[5]}$. If the patients don't get immediate treatment, the size of myocardial infarction will gradually expand, thus leading to arrhythmia, renal failure as well as shock. What's worse, the patients may probably get a sudden death when their conditions get worse, which exerts a great threat to their physical safety. The experimental results show that the total efficacy of research group is $97.78 \%$ and is much higher than that of $84.44 \%$ in control group.
In general, the incidence of adverse events happened in patients with acute myocardial infarction is gradually declining after percutaneous coronary intervention and is far lower than that of patients treated only by thrombolytic therapy. At the same time, when treated by percutaneous coronary intervention, the patients will be better treated and all their index of cardiac function will be improved, which is helpful to further control their treatment efficacy and improve their block in coronary artery ${ }^{[6]}$. As a doctor, it is necessary to choose appropriate treatment for the patients when treating. Only when we scientifically carry out our treatment, shall we better cure 
our patients who are suffering myocardial infarction.

Percutaneous coronary intervention is a form of operative treatment, which needs to perform coronary artery bypass operation for the patients when operating. It will use intracoronary stenting to further improve patients' conditions of myocardial infarction so as to achieve an effective adjustment to their conditions of myocardial infarction ${ }^{[7]}$. Meanwhile, it shall effectively improve their conditions of coronary artery stenoses by percutaneous coronary intervention so as to ensure the preservation and recovery of their cardiopulmonary function. In addition, by using percutaneous coronary intervention, it shall further improve their conditions of coronary arteriosclerosis and dredge their clogged artery so as to raise the dredge capability of the coronary artery and help improve patients' conditions. It can be find from clinic experiment that percutaneous coronary intervention had sound efficacy in treating patients with acute myocardial infarction ${ }^{[8]}$. It can quickly change the conditions of coronary artery stenoses and improve their cardiopulmonary function and cardiovascular supply, so as to get enough time for treatment. And after treatment, it shall effectively control the incidence of adverse events and improve patients' conditions of myocardial infarction.

In conclusion, we should actively apply percutaneous coronary intervention in the treatment of acute myocardial infarction so as to improve patients' cardiopulmonary function. Since its low incidence of heart and renal failure, safety and significant treatment efficacy in treatment, percutaneous coronary intervention bears great significance in clinic treatment.

\section{References}

1. Li X, Jin G. A Clinic Observation on emergency Percutaneous Coronary Intervention and Tirofiban combination treatment for Acute Myocardial Infarction (in Chinese). Journal of Critical Care in Internal Medicine 2017; 23(4): 315-317.

2. Buzure A. A clinic research on emergency Percutaneous Coronary Intervention for Acute Myocardial Infarction (in Chinese). Contemporary Medicine 2017; 23(15): 104-105.

3. Huang Y, Wang J. Comparison of clinic efficacy and safety between emergency percutaneous coronary intervention and intravenous thrombolysis therapy in the treatment of acute myocardial infarction (in Chinese). Practical Journal of Cardiac Cerebral Pneumal and Vascular Disease 2016; 24(7): 154155.

4. Yu H. Clinic efficacy of Urokinase Thrombolysis in 72 patients with Acute Myocardial Infarction (in Chinese). Medical Journal of Chinese People's Health 2018; 30(2): 156-158.

5. Pan L. Effect of Ticagrelor combined with Reteplase Thrombolytic Therapy on left ventricular systolic function and NT-proBNP level in patients with Acute Myocardial Infarction (in Chinese). Medical Journal of Chinese People's Health 2018; 30(12): 810.

6. Li T, Chen Y. Comparison of efficacy between Intravenous Thrombolysis and emergency Percutaneous Coronary Intervention in treating elderly patients with Acute Myocardial Infarction (in Chinese). The Journal of Medical Theory and Practice 2017; 30(13): 156-157.

7. Wu E, Cao Y. Clinical efficacy of emergency Intervention Therapy and Intravenous Thrombolysis in patients with Acute Myocardial Infarction complicated with Prehospital Cardiac Arrest (in Chinese). Guide of China Medicine 2018; 16(22): 91-92.

8. Kang H. Effect of psychological intervention on prognosis of emergency Coronary Intervention in Acute Myocardial Infarction (in Chinese). China Health Standard Management 2018; 9(20): 155-156. 